\title{
以“聚集诱导发光(AIE)”理论为载体开展化学类课程思政建设 的研究与实践
}

李恺, 何占航, 李中军 ${ }^{*}$, 藏双全 ${ }^{*}$

郑州大学化学学院, 郑州 450001

摘要: 聚集诱导发光(AIE)作为一种由中国科学家首先提出并被世界所公认的前沿科学理论, 为化学学科思政教育提 供了一个极佳的载体。通过对 AIE 理论和其发现者一一唐本忠院士科学研究事迹的学习, 可以使学生在了解科学前 沿进展的同时, 增强民族自豪感、激发学习热情, 为学生树立科研报国、永攀高峰的光辉典范。本文介绍了郑州大 学化学学院以“聚集诱导发光(AIE)”理论为载体开展化学类课程思政建设的研究实践和相关思考。

关键词: 课程思政; 聚集诱导发光; 教学案例

中图分类号: G64; O6

\section{Utilizing “Aggregation-Induced Emission (AIE)" Theory to Construct Course Ideology and Politics Teaching Case of Chemistry}

\author{
Kai Li, Zhanhang He, Zhongjun Li *, Shuangquan Zang * \\ College of Chemistry, Zhengzhou University, Zhengzhou 450001, China.
}

Abstract: As a frontier scientific theory, which is proposed by Chinese scientist and universally acknowledged, aggregation-induced emission (AIE) theory provides an ideal vehicle for constructing course ideology and politics teaching case of chemistry. By studying AIE theory and the story of Prof. Ben Zhong Tang, who is the discoverer of AIE theory, students' national pride and learning passion will be enhanced memorably. Meanwhile, Prof. Ben Zhong Tang is a glorious example for young students to serve the country and scale new heights. In this paper, the practices and thoughts of utilizing AIE theory to construct course ideology and politics teaching case of chemistry in college of chemistry of Zhengzhou University is introduced.

Key Words: Course ideology and politics; Aggregation-induced emission; Teaching case

“聚集诱导发光” (Aggregation-induced emission, 简称AIE)指的是一些含有特殊结构的分子, 其 在溶液分散状态下无光致发光而在聚集状态或固体状态下具有强烈光致发光性能的一种化学现 象 ${ }^{[1]}$ 。AIE现象由中国科学家唐本忠院士于 2001 年首先发现并报道, 经历了近 20 年的发展, AIE分子 已被广泛应用于有机光电材料、苂光探针、生物成像、过程监测等诸多领域, 成为国际公认的化学 科学研究的前沿热点之一 [2]。值得骄傲的是, 中国科学家在AIE领域的研究中一直处于国际领先水 平, 唐本忠院士也因在该领域的开创性贡献获得了2017年国家自然科学一等奖的殊荣。作为一种由 中国科学家首先提出并被国际所认可的前沿科学理论, AIE理论为化学学科思政教育提供了一个极 佳的载体。通过对AIE理论发展历程以及唐本忠院士科学研究事迹的学习, 可以增强学生的民族自

收稿: 2020-09-10; 录用: 2020-10-15; 网络发表: 2020-11-23

“通讯作者, Emails: lizhongjun@zzu.edu.cn (李中军); zangsqzg@zzu.edu.cn (藏双全)

基金资助: 郑州大学本科教育教学改革研究与实践项目(2020zzuJXLX002, 2020zzuJXLX062) 
豪感、激发学习热情, 为学生树立科研报国、永攀高峰的光辉典范。

郑州大学化学学院(原化学与分子工程学院)作为首批教育部高校 “三全育人” 综合改革试点院 (系), 在课程思政建设方面具有深厚的积累和丰富的实践经验。早在2017年, 郑州大学化学学院就已 经开始探索, 尝试把AIE理论作为课程思政元素引入本科教学 ${ }^{[3]}$ 。2019年, 郑州大学化学学院在本科 必修课 “综合化学实验” 中开设了 “水杨醛希夫碱的合成、表征及其聚集诱导发光性能” 综合实验。 该实验课融合科学前沿理论和课程思政元素, 引起了学生的热烈反响, 多位同学受该课程的影响进 入AIE相关领域开展研究学习和深造。结合学院特色, 我们进一步将AIE理论这一课程思政教育元素 拓展到理论课教学和实验课教学中, 打造了一个可复制、可推广、易移植、普适性好的课程思政精 品案例。本文介绍了郑州大学化学学院围绕AIE理论课程思政教学案例开展建设和进一步拓展课程 思政元素的一些思考。

\section{AIE理论作为课程思政案例引入教学的意义}

AIE理论是一个由中国科学家首先提出并发展成熟的原创的科学理论 ${ }^{[4]}$ 。通过对AIE理论的学 习, 有利于帮助学生厚植爱国情怀, 提高学生的民族自尊心和自豪感。同时, AIE理论至今仍在不断 完善, 其应用仍在不断拓展, 是化学学科十分活跃的前沿研究领域之一。通过学习AIE领域最新的进 展, 能有效激发学生的科研兴趣, 培养创新思维的同时提升其使命感和责任感。唐本忠院士在科学 研究中体现出的卓越品质, 为青年学生提供了光辉的榜样。他提倡 “见人皆所见, 思人所未思” 的 科研理念, 要求学生不但要有扎实的理论基础, 熟练掌握各种已有的知识, 更要具备敢于质疑经典、 突破传统理论的勇气和能力。“聚集诱导发光” 理论就是一个颠覆传统 “聚集诱导淬灭” 理论的创新 发现。唐本忠院士在科学研究中还特别强调团结合作, “Together We Shine, United We Soar!” 的协作 理念 ${ }^{[5]}$, 使中国在聚集诱导发光领域团结了一大批顶尖学者, 成功打造了国际高技术竞争中的中国 品牌。这些研究理念, 对初入科研的青年学生具有非常重要的启发作用, 有利于引导他们形成正确 的人生观和价值观, 培养良好的科研习惯。因此, AIE理论为化学学科思政教育提供了一个极佳的案 例, 通过对AIE理论发展历程、发现人事迹、前沿进展的深入挖掘, 可以体现丰富的思政教育意义, 使思想政治教育与专业教育同向同行、深度融合、相互促进。这也完美契合了教育部《高等学校课 程思政建设指导纲要》中对理科专业思政建设 “要在课程教学中把马克思主义立场观点方法的教育 与科学精神的培养结合起来, 提高学生正确认识问题、分析问题和解决问题的能力。理学类专业课 程, 要注重科学思维方法的训练和科学伦理的教育, 培养学生探索未知、追求真理、勇攀科学高峰 的责任感和使命感” 的要求 ${ }^{[6]}$ 。

\section{2 将AIE理论引入课程教学, 开展思政教育的实例}

在实际教学过程中, 郑州大学化学学院从理论课和实验课两方面同时入手, 在多个教学环节中 结合课程内容引入AIE理论, 融入课程思政元素, 拓展课程的理论深度和思想深度(图1)。目前, 已

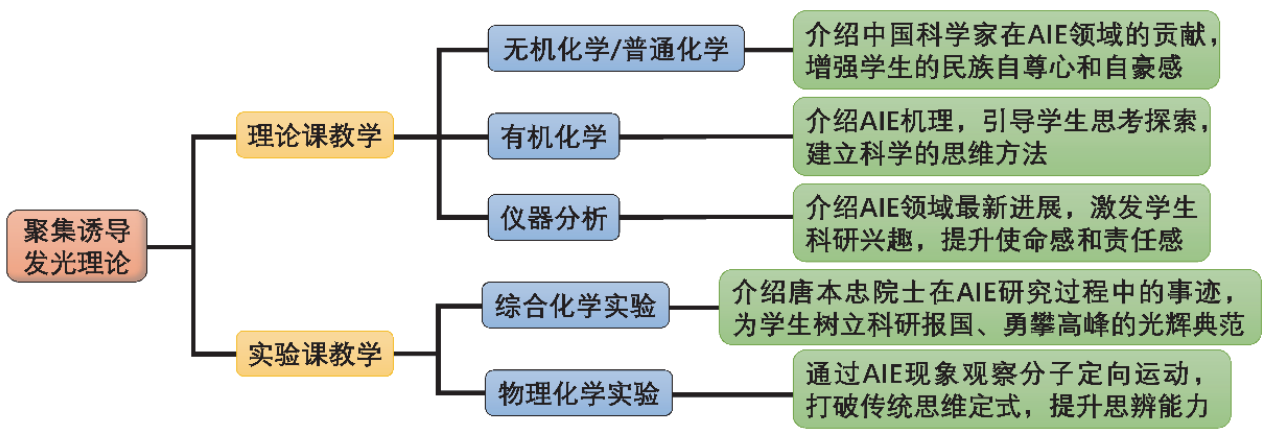

图1 郑州大学化学学院以AIE理论为载体开展的化学类课程思政建设 
在无机化学、普通化学、有机化学、仪器分析等多门理论课程中加入AIE理论相关案例, 并结合课程 内容融入不同的思政元素, 覆盖化学学院本科生基础必修课程和医学、化学工程、材料科学、生命 科学、物理学、环境科学等相关专业的化学类基础课程。同时, 在化学学院本科必修课 “综合化学 实验” 中开设了 “水杨醛希夫碱的合成、表征及其聚集诱导发光性能” 综合实验, 并计划从2021年 春季学期起, 在 “物理化学实验” 中开设 “利用聚集诱导发光现象观察分子的定向运动” 实验。通 过这两个实验, 使学生亲自动手实践, 了解AIE现象、原理和其前沿应用。

\section{1 在理论课教学中, 以AIE理论为载体展开思政教育的实例}

\subsection{1 无机化学/普通化学教学案例}

在化学专业一年级专业课无机化学和医学专业、化工专业、材料专业等一年级普通化学课程的 “分子结构” 相关章节中, 结合对 “分子间作用力” 和 “相似相溶” 原理的介绍, 设计课堂例题, 引入AIE的概念和特点。

例1 2001年, 中国科学家发现一种性质奇特的有机分子1-甲基-1,2,3,4,5-五苯基噻咯(化合物1), 结构如图2a所示。该分子在聚集状态或固体状态下表现出强烈的光致发光特性, 而分散在溶液中时 却没有光致发光性能, 这样的特性被称为聚集诱导发光, 简称AIE。请结合本课所学的知识, 判断该 分子在水和甲苯中光致发光性能的强弱, 并说明原因。

解: 从图 $2 \mathrm{a}$ 可知, 1 -甲基-1,2,3,4,5-五苯基噻咯是一个高对称性的有机分子, 其极性较小, 分子 间相互作用以范德华力为主, 这与甲苯分子间的相互作用力相似, 根据相似相溶原理, 1-甲基1,2,3,4,5-五苯基噻咯应易溶于甲苯, 处于分散状态, 在甲苯中无光致发光性能; 水分子是极性较大 的分子, 与 1 -甲基-1,2,3,4,5-五苯基噻咯分子间的相互作用力差别较大, 因此, 该分子在水中不易溶 解, 会形成聚集体, 表现出强烈的光致发光性能。

(a)

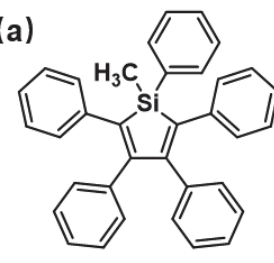

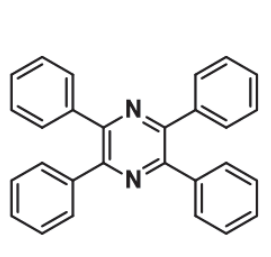

2

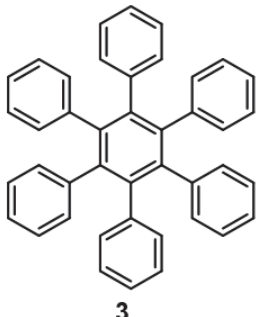

3

(b)

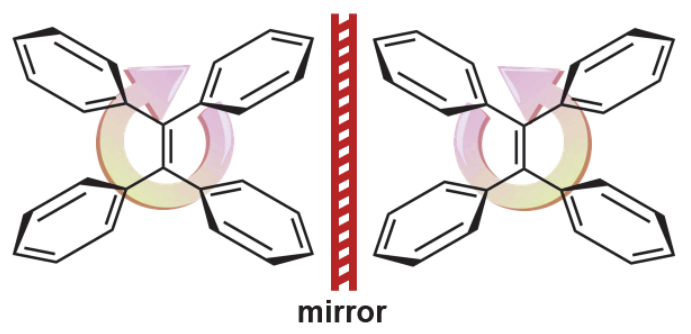

图2 (a) TPE在晶体中展现出的两种对映异构体; (b) 螺旋桨状AIE分子实例

在该例题讲授的同时, 教师可将思政元素进一步拓展, 介绍中国科学家在AIE领域的开创性贡 献, 强调AIE是一个由中国人首先提出并被国际广泛认可的科学理论, 增强学生的民族自尊心和自 豪感。

\subsection{2 有机化学教学案例}

在化学专业二年级专业课有机化学的 “立体化学” 相关章节中, 以具有 “螺旋桨状” 结构的AIE 分子为例, 讲解手性有机物结构。例如经典的AIE分子四苯乙烯(Tetraphenylethylene, 简称TPE), 其 所有的碳原子皆为 $s p^{2}$ 杂化碳原子, 表面上看应为平面共轭分子, 不具有手性。然而, 由于相邻苯环 
氢原子间的空间位阻作用, 其共轭体系不能处于同一平面, 从而导致了独特的 “螺旋桨状” 结构, 在不同手性溶剂中结晶出的晶体即可出现两种苯环向不同方向旋转排列的手性异构体(图2b) ${ }^{[7]}$ 。这 些例子可以帮助学生更好地理解有机分子手性和结构之间的关系。同时, 通过列举不同的 “螺旋桨 状” AIE分子并介绍其发展历程(图2a), 引导学生展开思考和探索, 尝试从理论上设计新型AIE分子。 通过这样的课程设计, 采用启发式教学, 使学生对所学理论的应用有直观感受的同时, 建立科学的 思维方法。

\subsection{3 仪器分析教学案例}

在化学专业三年级专业课仪器分析的 “荧光分析法” 相关章节中, 介绍AIE分子在荧光探针、生 物成像、诊疗一体化等方面的应用。经过近 20 年的发展, AIE材料已经成为一类极为重要的苂光材 料, 在化学、生物学、医学等领域被广泛应用 ${ }^{[8]}$ 。尤其值得骄傲的是, 中国科学家在这些研究中起到 了绝对的引领作用。教师在课堂上结合中国科学家报道的经典科研案例, 介绍AIE应用领域和应用 前景, 使学生了解AIE材料的最新发展, 激发他们对化学研究的兴趣。这样的课程设计, 使学生在接 触科学前沿热点的同时, 提升其科学研究的使命感和责任感。

\section{2 在实验课教学中, 以AIE理论为载体展开思政教育的实例}

\subsection{1 在综合化学实验课程中, 以AIE理论为载体展开思政教育}

2019年起, 我们在郑州大学化学学院三年级必修课程综合化学实验中开设了 “水杨醛希夫碱的 合成、表征及其聚集诱导发光性能” 综合实验。该实验利用苯酚和氯仿, 在碱性条件下通过ReimerTiemann反应合成水杨醛, 再利用所合成的水杨醛与水合肼反应, 得到具有AIE特性的水杨醛缩肼席 夫碱化合物。通过对水杨醛缩肼荧光性能的表征以及和经典荧光分子罗丹明6G苂光性能的对比, 让 学生了解光致发光的基本原理。作为一个专门实践AIE分子合成、展现AIE现象的实验, 该实验引起 了化学学院学生的热烈反响, 已有多位同学受该课程影响进入了AIE相关领域开展研究学习或继续 深造。在本课程中, 我们融入思政内涵, 通过讲授AIE现象的发现过程, 对比聚集诱导淬灭现象和聚 集诱导发光现象, 向学生传递 “见人所未见, 思人所未思” 的科研理念, 培养创新思维。通过讲解 AIE理论的发展历程, 将唐本忠院士在AIE研究过程中使命担当、勇于开拓、坚忍不拔的事迹在课堂 上进行详细的介绍, 为学生树立科研报国、勇攀高峰的光辉典范(图3)。该综合实验课程所需仪器设 备简单、易于推广, 相关实验在《大学化学》发表后得到了国内同行的广泛关注, 半年内已经下载 超过 360 次 ${ }^{[9]}$ 。

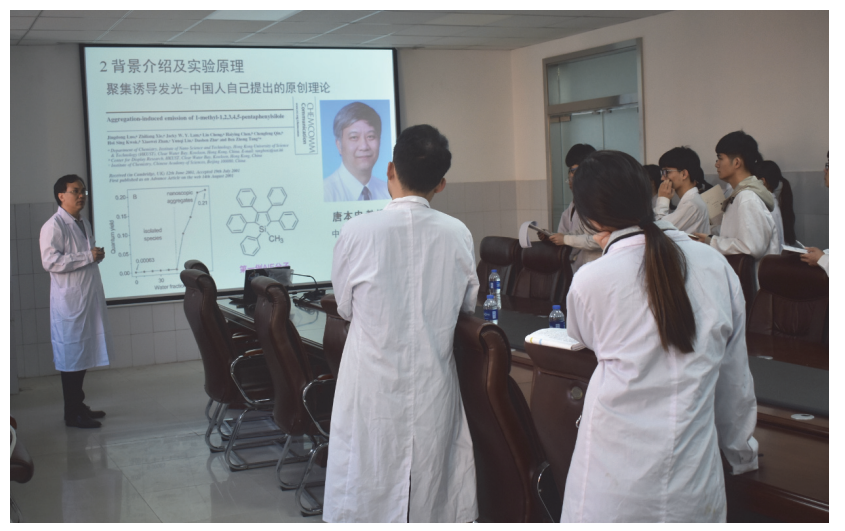

\subsection{2 以AIE理论为载体开展创新实验, 将思政元素引入物理化学实验课程教学}

2019年, 郑州大学化学学院设计了 “利用聚集诱导发光现象观察分子的定向运动” 的教学实验。 该实验将AIE、 “分子机器” 等科学前沿问题引入实验教学, 将苂光性能表征和化学动力学测试相结 
合, 提升学生利用课本知识解决实际问题的能力。利用荧光信号观察分子在固体状态下的运动规律 是2019年之后才逐渐发展起来的一项新兴技术 ${ }^{[10],}$, 该实验也是基于2019年最新报道的科研成果拓展 设计而成的 ${ }^{[11]}$ 。在该实验中, 学生利用4-丁氧基苯胺水杨酫席夫碱的AIE特性, 观察该分子在聚集状 态下研磨前后的苂光性能的变化, 探索其作为一种简单的具有自发定向运动能力的 “分子机器” 所 表现出的运动规律, 还可以进一步利用理论课所学的物理化学知识, 探索隐藏在该 “分子机器” 背 后的化学动力学和化学热力学原理。我们计划在2021年春季学期中, 将该实验引入化学专业必修课 程物理化学实验中。通过该实验让学生掌握化学动力学理论在研究实际问题中的应用方法, 同时展 示AIE研究领域的最新科研成果, 帮助学生打破思维定式, 提升思辨能力。

\section{3 进一步拓展课程思政元素的一些思考}

课程思政元素的引入方式, 除了理论课和实验课课堂教学之外, 还可以进一步拓展。例如, 在 由郑州大学承办的 “第33届中国化学奥林匹克(决赛)暨冬令营” 的比赛试题中, 题目6-4中将AIE思 政元素融入其中 ${ }^{[12]}$ 。在题干中, 向学生介绍 “聚集诱导发光现象是一种由中国科学家首先发现的光 致发光现象”, 提升学生民族自豪感, 促进其对化学的热爱。又如, 尝试将课程思政案例编入教材, 在郑州大学化学学院无机教研组主编的河南省十四五规划教材《无机化学原理》中, 将例 $\mathbf{1}$ 作为例题 编入其中, 该书已确定将于 2021 年由高等教育出版社出版。再如, 采用微视频等新手段将AIE思政元 素引入课堂教学。在设计 “利用聚集诱导发光现象观察分子的定向运动” 教学实验时, 我们曾有幸 邀请到唐本忠院士为团队进行指导并录制视频。以该视频作为素材, 我们进一步扩展制作了微视频, 在课堂上与学生分享, 让学生感受大师风采, 提高思政育人效果。

\section{4 结语}

在化学类课程教学中融入课程思政理念, 在润物无声、潜移默化中实现教育目标, 切入点十分 重要。选择合适的切入点, 进行深入思考和拓展, 开发可复制、可推广的课程思政案例是我们不断 的追求。本文介绍了郑州大学化学学院利用AIE理论为载体开展的课程思政教学案例, 分享了进一 步深化课程思政建设的思考, 希望能为相关高校开展化学类课程思政建设提供有益的参考。

\section{参 考 文 献}

[1] Hong, Y.; Lam, J. W. Y.; Tang, B. Z. Chem. Soc. Rev. 2011, 40 (11), 5361.

[2] Zhao, Z.; Zhang, H.; Lam, J. W. Y.; Tang, B. Z. Angew. Chem. Int. Ed. 2020, 59 (25), 9888.

[3] 李恺, 李媛媛, 藏双全, 唐本忠. 化学教育, 2017, 38 (22), 38 .

[4] Luo, J. D.; Xie, Z. L.; Lam, J. W. Y.; Cheng, L.; Chen, H.; Qiu, C.; Kwok, H. S.; Zhan, X.; Liu, Y.; Zhu, D.; et al. Chem. Commun. 2001, 1740.

[5] Mei, J.; Leung, N. L. C.; Kwok, R. T. K.; Lam, J. W. Y.; Tang, B. Z. Chem. Rev. 2015, 115 (21), 11718.

[6] 教育部关于印发《高等学校课程思政建设指导纲要》的通知. [2020-06-01].

http://www.moe.gov.cn/srcsite/A08/s7056/202006/t20200603_462437.html

[7] Jin, Y. J.; Kim, H.; Kim, J. J.; Heo, N. H.; Shin, J. W.; Teraguchi, M.; Kaneko, T.; Aoki, T.; Kwak, G. Cryst. Growth Des. 2016,16 (5), 2804.

[8] Qian, J.; Tang, B. Z. Chem 2017, 3 (1), 56.

[9] 李恺, 卢会杰, 李媛媛, 藏双全, 唐本忠. 大学化学, 2020, $35(1), 53$.

[10] Alam, P.; Leung, N. L. C.; Cheng, Y.; Zhang, H.; Liu, J.; Wu, W.; Kwok, R. T. K.; Lam, J. W. Y.; Sung, H. H. Y.; Williams, I. D.; et al. Angew. Chem. Int. Ed. 2019, 58 (14), 4536.

[11] Liu, J.; Xing, C.; Wei, D.; Deng, Q.; Yang, C.; Peng, Q.; Hou, H.; Li, Y.; Li, K. Mater. Chem. Front. 2019,3 (12), 2746.

[12] 展现化学之美、回归基础本源—2019全国高中生化学奥林匹克竞赛命题思路解析. [2020-04-29].

https://www.chemsoc.org.cn/popular/info/a3711.html 